\title{
L'optique des Voyages de Gulliver
}

Philippe HAMOU *

\begin{abstract}
Résumé : Contribution paradoxale à la querelle des Anciens et des Modernes, les Voyages de Gulliver proposent un message pessimiste sur la modernité et sur la nouvelle science qu'elle promeut: la simple sagesse et la juste mesure qui régnaient dans le monde ancien sont devenues des idéaux inaccessibles. Le monde moderne est inexorablement marqué par la disproportion, I'extension et la déclôturation de I'univers, la contingence et l'artificialité du point de vue humain sur le monde. Ce message philosophique est relayé dans le texte par un savant recours à I'optique des Modernes dans la construction de la fiction même des voyages : la perspective artificielle (Leon Battista Alberti) fournit un principe de relativité ; I'optique philosophique (George Berkeley) permet de rendre compte du caractère artificiel, construit par I'habitude, de notre connivence sensible avec le monde; I'optique instrumentale (le thème des yeux-microscopes, Robert Hooke et Nicolas Malebranche) suggère que l'univers auquel nous donnent accès la science et ses instruments n'est pas approprié à l'existence humaine.
\end{abstract}

Mots-clés : optique ; perspective ; yeux-microscopes ; modernité ; anthropologie philosophique ; Jonathan Swift ; Leon Battista Alberti.

Summary: Swift's masterpiece, Gulliver's travels, one of the most pregnant and paradoxical contributions to the Battle of Ancients and Moderns, offers a pessimistic view on modernity and modern science. Values of the ancient world such as wisdom, simplicity and good proportion have now become inaccessible ideals. The disproportion between man and his surroundings, the contingency and artificiality of his perspective on the world, the indefinite extension of the universe are inescapable features of modernity. This philosophical view is subtly expressed in Gulliver's travels narrative through the use of a threefold optical model: artificial perspective (Leon Battista Alberti) offers a principle of relativity ; philosophical optics (George Berkeley) accounts for the artificiality of our acquaintance with the visual world, showing that it is actually built out of habits and experience; instrumental optics

* Philippe Hamou, Université Paris-X, 200, avenue de la République, 92001 Nanterre. E-mail : philippe.hamou@u-paris10.fr 
(microscopic-eyes, Robert Hooke, Nicolas Malebranche) suggests that the universe to which science and its instruments give access is not fitted to human life.

Keywords : optics ; perspective ; microscopic-eyes ; modernity ; philosophical anthropology; Jonathan Swift ; Leon Battista Alberti.

Chacun connaît la fiction sur laquelle Jonathan Swift construit les deux premiers voyages de Gulliver. Un naufrage dépose le narrateur, Lemuel Gulliver, un brave chirurgien de vaisseau, sur une île inconnue qui se révèle bientôt peuplée d'hommes dont la stature est douze fois plus petite que celle des Européens. II ne s'agit pourtant pas de Pygmées ni de nains, car tout au royaume de Lilliput, plantes et animaux, à l'exception de l'encombrant voyageur, est à la mesure de ces créatures. À Brobdingnag, où Gulliver est abandonné lors d'un second voyage, la situation est inversée, les hommes, les plantes, les animaux ne sont géants qu'au regard du voyageur, qui apparaît à présent comme un minuscule et étrange animal, une sorte de caprice de la nature, qu'on ne parvient à bien voir qu'à l'aide d'une loupe.

Je ne crois avancer rien de bien nouveau en affirmant qu'un modèle optique régit ce dispositif fictionnel. En fait, on trouvait déjà une idée de ce genre dans la préface à l'une des toutes premières traductions françaises de Gulliver's travels, celle de l'abbé Desfontaines, parue en 1727 , un an à peine après l'original anglais ${ }^{1}$ :

"Les deux premiers voyages sont fondés sur l'idée d'un principe de physique très certain, savoir qu'il n'y a point de grandeur absolue, et que toute mesure est relative. L'auteur a travaillé sur cette idée et en a tiré tout ce qu'il a pu pour réjouir et instruire ses lecteurs, et pour leur faire sentir la vanité des grandeurs humaines. Dans ces deux voyages, il semble en quelque sorte considérer les hommes avec un télescope : d'abord il tourne le verre objectif du côté de I'œil, et les voit par conséquent très petits : c'est le voyage à Lilliput. II retourne ensuite son télescope, et alors il voit les hommes très grands : c'est le voyage à Brobdingnag. Cela lui fournit des images plaisantes, des allusions, des réflexions.»

1 - Jonathan Swift, Les Voyages de Lemuel Gulliver, trad. de I'abbé Desfontaines (Paris, 1727), 2 tomes en un volume, préface du traducteur. Sur I'abbé Pierre-François Guyot Desfontaines et sur cette traduction très infidèle, voir Sybil Goulding, Swift en France (Paris : Champion, 1924), chap. III. 
La comparaison de Desfontaines avec les instruments optiques réciproques que sont le télescope et le microscope ${ }^{2}$, sans doute suggestive, pèche par certains côtés et j'y reviendrai. Guère contestable en revanche est la proposition selon laquelle un principe de relativité est à l'œuvre dans les premiers Voyages et leur sert de fil conducteur. L'exacte caractérisation de ce «principe » mérite toutefois un moment $\mathrm{d}^{\prime}$ attention. II me semble en effet qu'il recouvre plusieurs dimensions et qu'il ne prend son sens véritable que dans le contexte plus large de ce que j'appellerai ici le dispositif optique des Voyages. Celui-ci, nous le verrons, sollicite trois thèmes distincts mais articulés : un thème perspectif, associant au principe strict de relativité des grandeurs un principe de proportion ; un thème psychologique - celui de l'habitude perceptive et des prisons sensorielles; et enfin un thème dioptrique et instrumental, celui des "yeux-microscopes». C'est seulement dans la conjonction des trois thèmes que le dispositif prend toute sa force et peut remplir véritablement les fins polémiques et philosophiques que Swift lui assigne.

\section{Le thème perspectif : relativité et proportions}

Le principe de relativité évoqué par Desfontaines trouve dans le récit lui-même une formulation explicite. C'est Gulliver qui le prononce au moment de sa première rencontre avec les géants : « [...] sans aucun doute les philosophes ont raison de nous dire que rien n'est grand ni petit que par comparaison ${ }^{3}$. » L'idée selon laquelle les grandeurs doivent s'apprécier de manière comparative peut s'entendre toutefois en deux sens. Dans le sens le plus immédiat, une même chose peut être dite tour à tour grande ou petite selon qu'on la compare à une autre chose particulière plus petite ou plus grande. C'est très certainement ce sens que vise Gulliver dans l'épisode considéré, car il se souvient alors avoir été géant chez les

2 - Desfontaines semble faire allusion au fait que certains des tout premiers microscopes construits au XVII ${ }^{\mathrm{e}}$ siècle n'étaient autre chose que des lunettes astronomiques montées à I'envers, I'objectif étant utilisé en guise d'oculaire. II semble que ce soit avec une telle lunette inversée que Galilée soit parvenu à voir dès les années 1610 des « mouches qui paraissent aussi grosses que des agneaux », d'après le témoignage du voyageur français Jean de Tarde - rapporté par Reginald S. Clay and Thomas H. Court, The History of the microscope (London : C. Griffin, 1932).

3 - Les Voyages de Gulliver, trad. de Maurice Pons, in Jonathan Swift, CEuvres (Paris : Gallimard, 1965), "Bibliothèque de la Pléiade » [ci-après Voyages], deuxième partie, chap. ${ }^{\text {er }}$, 97. Cf. Gulliver's travels, édition de Paul Turner (Oxford : Oxford Univ. Press, 1998) [ci-après $G$ 's travels], 74-75. 
Lilliputiens; c'est ce même sens encore qui prévaut lorsqu'il se demande un peu plus loin "si la race de ces mortels gigantesques elle-même n'apparaîtrait pas lilliputienne à son tour en quelque pays du monde que nous n'avons pas encore découvert ${ }^{4}$. » D'un point de vue simplement géométrique, toutes les grandeurs sont susceptibles d'être relativisées parce qu'il n'y en a pas une qui soit telle qu'on ne puisse en trouver de plus petites ou de plus grandes. Le grand ou le petit sont des propriétés relationnelles, non intrinsèques - ce sont des « accidents » qui résultent de la comparaison que I'on peut faire des grandeurs les unes aux autres. S'agissant de grandeurs géométriques, le principe est certes banal, mais appliqué comme il l'est ici aux grandeurs physiques et en particulier aux créatures vivantes, il reçoit une coloration assez typiquement moderne. L'idée ancienne qu'il existerait dans la nature des dimensions limites, au-delà ou en deçà desquelles la nature cesserait de produire des êtres capables de vie ou d'activité semble battue en brèche, depuis que les microscopes de Robert Hooke ou de Antonie Van Leeuwenhoek ont découvert aux hommes dans la moindre goutte d'eau des créatures innombrables, inimaginablement plus petites que le fameux ciron, qui passait jusqu'alors pour être "l'atome de la nature ${ }^{5}$. Bien avant qu'une théorie cellulaire ne vienne de nouveau fixer des bornes, le microscope ouvre ainsi aux modernes une perspective vertigineuse sur un possible emboîtement indéfini des dimensions de l'existence vivante. Nicolas Malebranche par exemple l'envisage de manière cocasse dans ce passage de la Recherche de la vérité ${ }^{6}$ :

"Les petits animaux dont nous venons de parler [les animalcules de Leeuwenhoek], ont peut-être d'autres petits animaux qui les dévorent, et qui leur sont imperceptibles à cause de leur petitesse effroyable, de même que ces autres nous sont imperceptibles. Ce qu'un ciron est à notre égard, ces animaux le sont à un ciron; et

4 - Ibid.

5 - Cf. Jules-César Scaliger, Exotericarum exercitationum (Paris, 1557), fo 263 : « [...] il est si petit qu'il ne paraît pas consister en atomes mais être un des atomes mêmes d'Épicure », cité par Robert Halleux, in Johannes Kepler, L'Étrenne ou la neige sexangulaire (Paris : J. Vrin, 1975).

6 - La Recherche de la vérité, in Nicolas Malebranche, CEuvres, I, éd. de Geneviève Rodis-Lewis (Paris: Gallimard, 1979), "Bibliothèque de la Pléiade», 55. Il y a dans I'application du principe de relativité aux créatures physiques une suggestion assez typiquement moderne qui s'appuie implicitement sur l'une des idées qui est au cœur de la révolution scientifique : l'extension et la divisibilité à l'infini de l'espace géométrique sont porteuses d'une signification physique. Elles suggèrent donc les possibles extension et divisibilité à l'infini de la matière elle-même. 
peut-être qu'il y en a dans la nature de plus petits et plus petits à l'infini, dans cette proportion si étrange d'un homme à un ciron. »

Ici, il semble simplement que Swift se soit plu à poursuivre dans les deux sens le jeu d'emboîtement des formes vivantes suggéré par Malebranche : les cirons ont peut-être des parasites qui les démangent, nous avons nous-mêmes des cirons, et passerions peut-être pour de la vermine pour quelques êtres plus considérables, lesquels à leur tour seront relativisés par d'autres géants, etc.

Il y a une seconde façon de comprendre l'idée selon laquelle l'exercice de la comparaison est crucial pour apprécier les grandeurs, et elle conduit, me semble-t-il, dans une direction toute différente. Cette seconde approche du principe de relativité consiste à dire qu'une chose apparaît grande ou petite lorsqu'on la compare, non pas à quelque autre chose plus petite ou plus grande, mais à l'idée de ce que devrait en être la taille «normale». Il ne fait guère de doute que le gigantisme ou le nanisme de Gulliver, envisagés du point de vue de ses hôtes, procèdent aussi de cette perspective sur la relativité. C'est parce que Gulliver est un homme et qu'il n'est pas une montagne ou un "splacknuck ${ }^{7}$ ", qu'il peut être dit trop petit ou trop grand : il ne répond pas à la norme de la stature humaine, et c'est cela même qui fait de lui un être déplacé, hors de " proportion ». À cet égard, il faut mettre l'accent sur le fait qu'il n'y a pas, dans les mondes de Lilliput ou Brobdingnag, de distorsion baroque des grandeurs, mais qu'on a affaire à chaque fois à des univers dont les parties constitutives sont proportionnées les unes aux autres et généralement conformes aux proportions « normales» qui règnent dans l'ancien monde. C'est un point que Gulliver lui-même confirme maintes et maintes fois, se plaisant à calculer - une fois donnée la taille des hommes, qui est en quelque sorte l'index de toutes les autres - celle des maisons, des villes, des plantes ou des animaux, voire la "contenance » volumique de I'Homme Montagne qui, étant 12 fois plus grand qu'un Lilliputien, devra manger 1728 fois plus ${ }^{8}$. Conséquence de ce strict respect des proportions, la seule manière dont peuvent s'établir la petitesse des Lilliputiens ou la grandeur des géants est extrinsèque : il faut pour cela le regard de Gulliver et la conscience, à la fois perceptive et mentale, qu'il peut avoir des dimensions de son propre corps. A

7 - «Une petite bête de ce pays, très jolie à voir et longue de six pieds » (soit longue comme un homme adulte). Cf. Voyages, II, 2, p. 107 et $G^{\prime}$ s travels, 85.

8 - Voyages, I, 3, p. 55-56; G's travels, 31. 
contrario, si Gulliver lui-même rétrécissait ou grandissait à la faveur de ses naufrages, il n'y aurait aucun moyen pour lui, comme pour nous, de savoir qu'il est entré dans un autre monde.

Ceci indique quelque chose d'important sur la notion de norme ou de juste proportion : la norme se définit de manière immanente, comme résultant d'un système de relations internes régissant un monde. Une chose est normale si elle s'inscrit harmonieusement dans ce système, déplacée ou aberrante si elle y échappe. II est à noter que ce qui donne au rapport relatif des grandeurs du monde dans lequel nous vivons son privilège normatif n'est pas seulement I'habitude (contingente) que nous avons de ces rapports, mais la conviction latente que ces rapports sont préférables à tous les autres, s'insèrent dans un cadre harmonieux et final. Ce point de vue reste en tout cas celui des débuts de l'âge moderne, dominés encore par l'idée traditionnelle d'une théologie naturelle centrée sur l'homme, où l'on conçoit que l'ordre et l'agencement du monde soient le résultat d'une volonté finale ou providentielle. L'univers est tourné vers l'homme et son usage en vertu de ce qu'on pourrait appeler, avec Emmanuel Kant, une " finalité externe de la nature ». L'herbe n'est ni trop haute ni trop basse, les fruits sont à portée de main et comestibles, les animaux domesticables : tout a été voulu de manière finale pour être à la mesure et au service des hommes. En conséquence, et réciproquement, I'homme peut être dit la mesure de toute chose : c'est à partir de l'étalon que fournit la stature humaine que s'établissent les unités de mesure, comme les pieds et pouces, c'est à partir de ses besoins que se définissent l'utile et le nuisible, le proche et le lointain. L'homme enfin est lui-même comme un microcosme, ses parties ou ses organes étant proportionnés les uns aux autres de manière harmonieuse et conforme aux proportions de l'ordre cosmique.

On notera que le thème humaniste des proportions humaines et de leur harmonie fait une discrète apparition dans le premier Voyage. Gulliver y explique la manière dont les couturières lilliputiennes ont réussi à faire son costume avec une parfaite exactitude, en ne prenant pas plus qu'une ou deux mesures car elles ont appliqué le principe " deux tours de pouces valent un tour de poignet et ainsi de suite jusqu'au tour de cou et de taille ${ }^{9} »$. Le costume de Lilliput doit évidemment être comparé avec l'habit mal taillé que le tailleur mathématicien de Laputa fait à Gulliver, au terme d'un relevé

9 - Voyages, I, 6, p. 73-74; G's travels, 51. 
mathématique absurdement compliqué ${ }^{10}$. Le contraste révèle que les mathématiques modernes sont du côté de la pure relativité, et ne visent pas l'utilité comme une fin en soi, tandis que les principes harmoniques mis en œuvre par les couturières de Lilliput appartiennent encore à l'ordre de la science utile, et procèdent de la reconnaissance des rapports harmoniques présents dans la nature.

Je reviendrai plus loin sur cette importante tension entre les deux principes de relativité, mais il importe ici de bien comprendre leur lien dans la construction de la fiction des Voyages. Un rapprochement suggestif peut être fait avec la perspective picturale où relativité et proportion fonctionnent conjointement pour expliquer la « vraisemblance » optique d'une composition. L'idée de base est la suivante : en dépit du fait que la perspective représente en petit ce qui est grand (mais censé être lointain), on peut toujours apprécier la vraisemblance optique d'une composition picturale en comparant la grandeur des objets qui sont représentés comme mitoyens ou sur un même plan, et en utilisant comme norme ou critère d'appréciation l'idée que nous avons d'une grandeur étalon, modèle et norme pour toutes les autres - cette grandeur étant généralement celle de la stature humaine ${ }^{11}$. Ce jeu conjoint de la relativité et de la proportion est parfaitement explicite dans ce passage du De pictura de Leon Battista Alberti ${ }^{12}$ :

"Il faut ajouter à cela l'opinion des philosophes qui affirment que si le ciel, les astres, les mers, les montagnes, les êtres vivants euxmêmes et tous les corps étaient par la volonté des dieux réduits à la moitié de ce qu'ils sont, peut-être ne nous paraîtraient-ils aucunement plus petits que ce qu'ils sont maintenant. Car ce qui est grand, petit, long, court, haut, large, étroit, clair, obscur, lumineux, ténébreux, et généralement tout ce qui peut aussi bien être ou ne pas être dans les choses, les philosophes les nomment accidents et ils sont tels, en effet, que leur connaissance ne se fait que par comparaison. Virgile dit qu'Énée dépasse tous les hommes des épaules, mais si on le compare à Polyphème, il semblera un pygmée. On rapporte qu'Euryale était très beau, mais comparé à Ganymède

10 - Voyages, III, 2, p. $171 ;$ G's travels, 152.

11 - Les auteurs de l'optique ancienne, tel Alhazen, avaient déjà souligné l'importance de tels jugements tacites dans l'appréhension visuelle des grandeurs et distances (cf. The Optics of Ibn al-Haytham [Alhazen], liv. I-III, éd. et trad. anglaise d'Abdelamid I. Sabra (Londres: Warburg Institute, 1989), liv. II). Sur les liens entre I'optique médiévale et la perspective albertienne, voir en particulier Gérard Simon, Optique et perspective, d'Ibn al-Haytham à Alberti, dans Id., Archéologie de la vision (Paris : Le Seuil, 2003).

12 - Leon Battista Alberti, De la peinture. De pictura (1435), éd. latine et trad. française de Jean-Louis Schefer (Paris : Macula Dédale, 1992), 111-113. 
enlevé par les dieux, sans doute semblerait-il laid [...]. Il y a dans le fait de comparer les choses une force qui nous aide à comprendre le plus, le moins ou l'égalité qui est en elles. Ainsi disons-nous grand ce qui est supérieur au petit, très grand ce qui est supérieur au grand, lumineux ce qui est plus lumineux que le clair. La comparaison se fait donc d'abord avec les choses très connues. Et comme I'homme est pour I'homme la plus connue de toutes les choses, peut-être Protagoras, lorsqu'il disait que I'homme est la mesure et la règle de toute chose, entendait-il que I'on pouvait précisément connaître les accidents de toutes les choses en les comparant à ceux de l'homme. Tout cela nous permet de comprendre que, aussi petits soient les corps dans la peinture, ils paraîtront grands ou petits par comparaison avec I'homme qui y sera peint. De tous les Anciens, celui qui semble avoir le mieux saisi cette force de la comparaison est le peintre Timanthe, qui, dit-on, en peignant sur un petit panneau un Cyclope endormi fit à côté de lui des satyres étreignant le pouce du dormeur pour que par cette comparaison le dormeur parût encore plus grand. »

Ce texte méritait d'être cité tout au long car il entretient des affinités surprenantes avec Les Voyages ${ }^{13}$, la plus évidente étant la référence au Cyclope représenté par Timanthe entouré de satyres lui étreignant le pouce. L'image est une préfiguration saisissante de Gulliver endormi sur la plage de Lilliput. Une autre affinité notable est la mention, quasiment dans les mêmes termes, de l'appréciation des «philosophes » selon laquelle les accidents, comme le grand ou le petit, ne sont connus que par comparaison. Si I'on peut penser ici à Platon et surtout Aristote ${ }^{14}$, une autre référence philosophique, plus mystérieuse, ouvre le texte, à savoir cette " opinion des philosophes qui affirment que si le monde et nous-mêmes étions réduits de moitié par un décret des dieux, peut-être que les choses ne nous apparaîtraient en aucune façon changées ». Â ma connaissance, les divers éditeurs du De pictura sont toujours restés silencieux ou évasifs sur la source probable de cette « opinion ». Selon toute probabilité, Alberti I'emprunte à un traité du XIV siècle, la

13 - Je ne suis pas en mesure cependant d'établir que Swift connaissait le De pictura dont il n'existe pas de traduction anglaise au début du XVIII ${ }^{\mathrm{e}}$ siècle.

14 - Par exemple, Métaphysique, 1026b : « [...] I'accident n'a, en quelque sorte, qu'une existence nominale. » Les éditeurs de Swift mentionnent généralement George Berkeley pour éclairer la référence à la maxime des philosophes dans le texte des Voyages (cf. supra, n. 3). Mais les propos de Berkeley sur la relativité des grandeurs n'ont rien d'original. Le texte d'Alberti suffit à attester que l'idée a une origine plus ancienne. 
Questio de apparentia rei ${ }^{15}$, un ouvrage généralement attribué à Nicole Oresme, dont des copies manuscrites circulaient assez largement en Italie vers la fin du Moyen Âge. En tout état de cause l'expérience de pensée qui est imaginée ici ne manque pas d'entrer en résonance avec le procédé swiftien : les mondes possibles et parallèles de Lilliput et Brobdingnag sont de facto l'illustration fictionnelle du décret divin : si ce n'était la présence dérangeante de Gulliver, rien en eux ne révélerait leur disproportion au regard de notre monde. J'ajouterai un dernier mot sur la troisième référence philosophique de ce texte, la fameuse sentence de Protagoras («L'homme est la mesure de toutes choses 16 »), laquelle, me semble-t-il, est détournée de son sens relativiste et sophistique - il n'y a de vérité que dans le point de vue de chacun - pour acquérir un sens typiquement humaniste - l'homme est le bon point de vue et le bon étalon pour considérer l'ordre que Dieu a mis dans les choses. On est là devant un phénomène assez typique de l'histoire des idées : un motif ancien est investi d'un sens nouveau tout en conservant l'autorité que lui donne son ancienneté. L'homme est la mesure de toute chose, cela signifie pour Alberti, non seulement que la représentation des figures humaines est l'index des grandeurs représentées sur le tableau, et du même coup le garant de la vraisemblance perspective; mais que I'homme est également le pourvoyeur des justes ou bonnes proportions du tableau parce que lui-même, fait à l'image de la divinité, est harmonieusement proportionné. Il y a ainsi un élément de normativité esthétique dans le thème des proportions humaines, élément qui est encore très présent dans la première théorie perspective (celle d'Alberti, de Dürer) mais qui finira par disparaître chez les théoriciens ultérieurs.

La proximité entre le procédé swiftien et ce schème albertien est évidente et permet de tirer une première conclusion au sujet du dispositif optique des Voyages : les deux premiers voyages exhibent de manière presque caricaturale et en tout cas obsédante le principe qui fonde la vraisemblance de ce qu'Alberti appelait une

15 - Manuscrit, Venise, Biblioteca Marciana, VI, 72, fos 155rb-162vb. Les recherches que Jean Celeyrette a consacrées à ce texte (cf. son « Apparence et imagination chez Nicole Oresme », publié ici-même) ont attiré mon attention sur cette filiation hautement vraisemblable et méconnue. La Questio avance en effet une expérience de pensée affirmant que si tous les corps sensibles étaient diminués dans le rapport de 2 mais qu'une diminution corrélative de nos organes nous conduisait à percevoir des apparences multipliées par 2, la diminution nous resterait imperceptible, et, si cette diminution était continue, elle resterait non perçue jusqu'au moment final de l'annihilation.

16 - Cf. Platon, Théétète, 152a. 
historia, une scène représentée en perspective. Ce principe, pseudo-antique mais en fait typiquement humaniste, affirme en substance: I'homme est la mesure des choses, le monde à la mesure des hommes. Je crois qu'on a là déjà une première indication sur les intentions philosophiques de Swift, car les Voyages dans leur ensemble tournent assurément autour de ce dictum, I'interrogent et demandent s'il est encore énonçable ou soutenable dans le monde nouveau de la science, un monde qui semble avoir ôté à l'homme son privilège pour le plonger dans le vertige des deux infinis.

\section{Le schème du dépaysement et de l'habituation perceptive}

Plusieurs allusions dans les voyages révèlent que Swift avait au moins une teinture d'optique moderne, et qu'il avait retenu de la Nouvelle théorie de la vision de George Berkeley - son compatriote et ami - l'idée selon laquelle notre familiarité " optique » avec le monde n'est pas native mais qu'elle s'est construite avec l'expérience, sur la base de l'habitude et de la suggestion. La thèse, en un sens, n'est pas spécifique à Berkeley. On en trouvait déjà les prémisses chez John Locke et chez William Molyneux qui considéraient l'un comme l'autre qu'un aveugle-né rendu à la vue ne pourrait pas distinguer immédiatement la différence des figures et des distances avant d'avoir appris à lier ses idées tactiles et ses idées visuelles ${ }^{17}$. Berkeley radicalise ce point de vue en affirmant que la grandeur, la distance, la situation non seulement ne sont pas des objets immédiats de la vision mais ne sont pas des objets visuels du tout. C'est la conjonction constante de certaines impressions tactiles ou kinesthésiques avec des impressions visuelles (de lumière et de couleur) qui finit par instituer ces dernières comme des signes pour les premières ${ }^{18}$. Cette conjonction dépend de l'existence d'un ordre institué, un "cours régulier» de la nature. Si nous étions plongés dans une autre sorte de nature, nos impressions visuelles pourraient être pourvues d'une autre interprétation spatiale, associées à d'autres impressions tactiles ou peut-être même totalement dissociées d'elles.

17 - Cf. John Locke, An essay concerning human understanding, $2^{\mathrm{e}}$ éd. (Londres, 1694), liv. II, chap. 9.

18 - Cf. George Berkeley, An essay toward a new theory of vision (Dublin, 1709), § 45, 147. 
Swift, très certainement, joue avec ce thème, même s'il ne le tire pas jusqu'à son extrémité métaphysique ${ }^{19}$. Il s'en sert pour rendre compte tout à la fois des effets du « dépaysement » optique procuré par le changement d'échelle et de la dynamique d'habituation qui se met en place au fil du temps passé dans les contrées imaginaires. Le premier point est en fait l'un des principaux ressorts de la satire swiftienne. Si les Voyages sont dépaysants, ce n'est pas parce que les contrées visitées sont exotiques. Le dépaysement vient seulement du fait que le « préjugé » optique sur les grandeurs est rompu. Les objets les plus familiers, un rasoir, une montre à gousset, un peigne, tirés de la poche de Gulliver et décrits par les Lilliputiens, restent (en dépit de leur description scrupuleuse) totalement méconnaissables et irréels ${ }^{20}$. Réciproquement, Gulliver, observateur des mœurs locales, s'indigne et s'émeut devant les comportements absurdes, les institutions bancales, etc. de ses petits hôtes, sans jamais percevoir l'étrange proximité que sa micrographie présente avec le tableau qu'un acerbe pamphlétaire pourrait dresser de son Angleterre natale. Ainsi, accepter la fiction des Voyages, c'est reconnaître que notre familiarité avec le monde repose sur des préjugés optiques, des habitudes psychologiques, des conventions morales. Par le jeu de la relativité optique ou de la mise à distance, Swift redonne une figure visible à ce qui, dans I'accoutumance sociale, était devenu invisible, éveillant chez son lecteur une indignation salutaire.

Mais tandis que le lecteur est invité à partager les révoltes de Swift, Gulliver, lui, fait son chemin sur un tout autre plan. II ne maîtrise pas I'interprétation morale de son dépaysement, pas plus que l'aveuglené opéré ne comprend tout d'abord ce qui lui arrive. La rupture de familiarité, il la vit comme une perte de soi et non comme un accès à la lucidité. Tout pour lui est à conquérir. Une langue aux sonorités impossibles, un univers perceptif incongru, un statut social, une identité. La supériorité de jugement que pourrait lui donner sa

19 - Chez Berkeley, le traitement de la sensation comme un langage permet de préparer le terrain à l'immatérialisme - je défends cette thèse en détail dans mon article : Sens et fonction du modèle linguistique dans la nouvelle théorie de la vision, in Dominique Berlioz (éd.), Berkeley, langage de la perception et art de voir (Paris : PUF, 2003). Swift, qui admirait l'intelligence de Berkeley, considérait néanmoins avec quelque ironie son " immatérialisme », la doctrine selon laquelle toute la réalité sensible est constituée par nos idées. II écrit ainsi à John Arbuthnot, en 1714 : « Le pauvre philosophe Berkeley a maintenant l'idée de la santé, qui a beaucoup tardé à naître en lui. Car il avait si fortement ancrée l'idée d'une fièvre maligne, que celle-ci n'a cédé que très difficilement la place à son contraire » (cité et traduit dans Swift, OEuvres..., op. cit. in n. 3, 1604).

20 - Voyages, I, 2. 
situation d'étranger, de Huron, il ne peut en profiter, car pour comprendre le nouveau monde dans lequel il est plongé, il lui faut $s^{\prime} y$ insérer et, à mesure qu'il s'y insère, il perd le pouvoir de se mettre à distance. Ainsi, il finit par être impliqué à Lilliput dans un réseau de cabales et d'insinuations contre lequel il est beaucoup moins bien armé qu'il ne paraît ${ }^{21}$. De même, chez les géants, il prend peu à peu l'habitude de voir tout en grand au point que, libéré par les hommes de sa taille, il ne peut s'empêcher de les trouver ridiculement petits et sans force ce qui lui vaut de passer pour un dément ${ }^{22}$.

Ainsi, à mesure que Gulliver éduque ses sens dans l'univers des géants ou dans celui des Lilliputiens, I'image mentale de son propre moi s'agrandit ou se rétrécit à son insu. Comme on le voit, ce second versant du modèle «psychologique » de la théorie de la vision déplace le foyer de la satire. Le motif du dépaysement optique fonctionnait à peu près comme celui de l'ingénu ou du bon sauvage, il permettait de mettre à nu les travers du monde, en les exposant avec l'ingénuité de celui qui décrit sans comprendre ni participer. Mais le thème réciproque de « I'habituation » perceptive produit d'autres effets. II révèle le rôle de la suggestion inconsciente et de l'expérience commune dans la constitution de l'image que nous nous faisons de nous-mêmes, et la difficulté qu'il y a à faire coïncider cette image avec celle que renvoient de nous nos prétendus «semblables ». Il y a dans ce dernier point je crois l'un des motifs les plus profonds et personnels de l'écriture swiftienne.

\section{Microscopes et yeux-microscopes}

Un troisième thème optique nous ramène au propos de Desfontaines qui comparait la double fiction des voyages à l'usage successif par Gulliver d'un microscope et d'un télescope. Il y a certainement quelque chose d'assez juste et fort dans cette comparaison, à condition cependant de l'entendre dans un sens très général. On peut dire en effet qu'avec la fiction des Voyages, Swift referme une boucle métaphorique ouverte au début du siècle lorsqu'on commença à comparer les découvertes télescopiques de Galilée à celles du « Nouveau Monde » par Christophe Colomb ou Amerigo Vespucci, ou lorsque, avec Robert Hooke et Joseph Glanvill, on

$21-$ Ibid., l, 6 et 7.

22 - Ibid., II, 8, p. 154 ; G's travels, 139. 
pressentit, dans les découvertes du microscope, une " america of secrets », " an unknown Peru of Nature » 23. Ainsi, tandis que les savants crient «Terre ! » en contemplant I'univers grossi ou agrandi dans leurs lunettes, Gulliver fait très exactement le mouvement réciproque : en naviguant sur des mers inconnues, ce n'est pas une Amérique qu'il découvre mais bien une sorte de "dioptrical world », selon l'expression parlante de Robert Boyle.

La métaphore toutefois reste à préciser et la manière dont Desfontaines tente de le faire, associant le télescope à Brobdingnag et le microscope à Lilliput, n'est guère convaincante. S'il est en effet possible de trouver des indices textuels du fait que Swift avait bel et bien à l'esprit le modèle du microscope dans la fiction des deux premiers voyages, il est plus difficile d'appliquer celui du télescope au second : les géants ne sont pas des êtres lointains pour lesquels une lunette d'approche serait nécessaire. II semble plutôt que I'inversion à laquelle procède Swift ne soit pas celle de l'instrument, mais celle, plus subtile, qui consiste à substituer l'observateur à l'observé. Scrupuleux micrographe à Lilliput, Gulliver devient luimême à Brobdingnag, une de ces créatures minuscules qu'on doit observer à la loupe, sans d'ailleurs parvenir à en bien comprendre la nature. Ce fait traduit aussi le changement de registre de la satire : même si Gulliver reste le narrateur, le lecteur est invité à changer de point de vue, et à cesser de regarder les choses seulement avec les yeux de Gulliver, pour porter sur Gulliver lui-même le regard tour à tour savant, moqueur ou incrédule du géant. La scène où Gulliver est examiné en séance par les savants du royaume, parodie transparente des débats de la Royal Society sur les diverses curiosités de la nature produites en séance par le curator of experiments, en est peut-être l'exemple le plus savoureux ${ }^{24}$ :

«Sa Majesté fit mander les trois grands savants qui, selon la coutume locale, étaient de semaine au Palais ${ }^{25}$. Ces messieurs, après $\mathrm{m}^{\prime}$ avoir examiné en détail avec un soin minutieux, furent d'opinions différentes à mon sujet. Tous s'accordèrent à déclarer que je ne pouvais avoir été produit selon les lois normales de la nature, puisque j'étais dépourvu des qualités nécessaires à la

23 - Cf. Joseph Glanvill, Plus ultra (Londres, 1668).

24 - Voyages, II, 3, 113-114. II n'est guère besoin ici de souligner l'ironie : admettre, comme le font les empiristes, le "caprice de la nature», pour éviter les causes occultes d'Aristote, c'est recourir tout aussi bien à un asile d'ignorance.

25 - La traduction de Pons gomme malencontreusement I'allusion aux réunions hebdomadaires (weekly meetings) instituées à la Royal Society. 
conservation de la vie, ne pouvant ni grimper aux arbres, ni fuir rapidement ni creuser le sol pour $\mathrm{m}^{\prime} \mathrm{y}$ cacher... Après de longs débats, ils conclurent à l'unanimité que je n'étais rien qu'un relplum scalcath - mot à mot lusus naturae - définition qui concorde avec les doctrines actuellement à la mode en Europe, car ceux qui les professent, dédaignant de faire appel, pour éluder les difficultés, à la théorie ancienne des causes occultes, qui ne permettait guère aux disciples d'Aristote de dissimuler leur ignorance, ont inventé cette admirable solution passe-partout, pour le plus grand avancement du savoir humain. »

De même, Gulliver, faisant l'éloge des institutions anglaises et des guerres, devient sous le regard du bon et grand roi de Brobdingnag, un représentant de « la plus effroyable vermine à qui I'on ait permis de ramper à la surface de la terre 26 ». Le thème de la vermine microscopique ressurgit d'ailleurs en écho lorsque Gulliver est amené à voir les poux géants sur les mendiants de Brobdingnag, animaux qu'il se plaît à décrire avec force détails, à la manière de Hooke dans sa Micrographia :

" Je distinguais les pattes des poux à l'œil nu, bien plus clairement qu'on ne les voit chez nous au microscope, et le groin avec lequel ils fouillaient comme des cochons. Je n'en avais jamais vu auparavant et il m'eût intéressé d'en disséquer un, si j'avais eu les instruments adéquats [...]. Ils étaient pourtant si horribles à voir que j'en avais la nausée ${ }^{27}$. »

De façon générale, une référence tacite au microscope est rattachée dans les Voyages à ce que l'auteur décrit comme une forme d'intensification de la sensorialité, phénomène présenté $d^{\prime}$ ailleurs comme assez dégradant et dégoûtant : le spectacle de la reine qui mange son repas est littéralement "répugnant »; Gulliver perçoit sur un grain de beauté d'une géante de la Cour un poil de la grosseur d'une ficelle ; il a du mal à supporter l'odeur intense des jeunes filles de la Cour qui se dénudent sans façon devant lui 28 .

On notera qu'en prêtant ici à Gulliver une vue perçante qui lui permet d'appréhender un univers grouillant et hideux qui échappe totalement à l'œil des géants, Swift se conforme à un autre motif philosophique classique associé à l'usage des microscopes, le

26 - Voyages, II, 6, p. $142 ; G^{\prime}$ s travels, 126.

27 - Voyages, II, 4, p. 122. On comparera aux descriptions du pou données par Robert Hooke dans sa Micrographia (Londres, 1665), observ. LIV "Of a louse », et schéma XXXV.

28 - Voyages, II, 5. 
thème des "yeux-microscopes», tel que l'exposait par exemple Malebranche 29 :

\begin{abstract}
"Mais si nous avions les yeux faits comme des microscopes, ou plutôt si nous étions aussi petits que les cirons et les mites, nous jugerions tout autrement de la grandeur des corps. Car sans doute ces animaux ont les yeux disposés pour voir ce qui les environne, et leur propre corps beaucoup plus grand ou composé d'un plus grand nombre de parties que nous ne le voyons : puisque autrement ils n'en pourraient pas recevoir les impressions nécessaires à la conservation de leur vie, et qu'ainsi les yeux qu'ils ont, leur seraient entièrement inutiles. »
\end{abstract}

Le thème des yeux-microscopes réapparaît chez Locke et Berkeley et joue un rôle particulier chez ces philosophes pour mettre en évidence l'idée selon laquelle les sens dont nous disposons sont formés de manière providentielle pour nous livrer le visible qui est adapté à nos besoins ${ }^{30}$. Comme l'explique Berkeley, si nos yeux étaient transformés en microscopes, nous ne gagnerions guère au change, nous perdrions la connexion providentielle du visible et du tangible et serions abandonnés à la " vaine distraction de voir ${ }^{31}$ ". Cette impuissance de fait est bien celle de Gulliver qui ne doit sa survie dans le monde hostile qui l'entoure, plein de méchantes bestioles et d'à-pics vertigineux, qu'aux bons soins et à la vigilance de Glumdalclitch, I'enfant géant. Ici encore, on peut dire que Swift, en ramenant Gulliver aux dimensions d'une mite voyante, suit une suggestion berkeleyenne ou malebranchiste explicite, mais il lui donne un tour assez idiosyncrasique : le monde microscopique n'est pas seulement un autre monde, inadapté à nos besoins d'hommes, et dans lequel, si nous y étions plongés organiquement, nous ne pourrions pas réellement agir ou survivre ; c'est un monde empli de visions d'horreurs, de monstres épouvantables et d'odeurs innommables. On notera que l'idée contraste ironiquement avec un thème qui, depuis Malebranche, fait florès chez les microscopistes : celui de la beauté de l'infime, la considération de la surabondante providence divine qui s'y manifeste et qui conduira les observateurs du début du XVIII ${ }^{\mathrm{e}}$ siècle (comme Jan Swammerdam) à

29 - Malebranche, op. cit. in n. 6, liv. I, chap. 6, 58.

30 - Locke, op. cit. in n. 17, II, 23, p. 12.

31 - Berkeley, op. cit. in n. 18, § 85-86. 
faire du petit insecte le cœur d'une sorte de mystique physico-théologique ${ }^{32}$.

\section{« L'optique » philosophique des Voyages : une critique de l'éthos scientifique des Modernes}

Les microscopes, au début du XVIII ${ }^{\mathrm{e}}$ siècle, font partie de la panoplie de la science nouvelle, anatomisante et expérimentale, ils en sont même, à certains égards, des instruments symboliques. Aussi, il y a lieu de croire que la critique implicite qu'on voit se dessiner à leur sujet, dès le second voyage, a, aux yeux de Swift comme de ses contemporains, une portée élargie. Peut-être la science est-elle, comme le microscope qui la représente, myope : le gain de savoir qu'elle nous apporte doit être mis en balance avec une perte correspondante de la conscience de la totalité, du sens de l'ordre et de la juste disposition des priorités.

Comme on le sait, la science des Modernes, et particulièrement cette natural experimental philosophy qu'on pratique à la Royal Society fait l'objet d'une charge violente dans le troisième voyage, celui qui conduit Gulliver dans I'île de Laputa et le met en contact avec ses savants et ses académies. La figure des savants de Laputa, " un œil tourné vers le dedans, l'autre fixé sur le zénith ${ }^{33}$ », donne déjà la direction d'ensemble de la critique. Pour ces hommes, seuls comptent l'intériorité et les lointains. L'intériorité est l'espace où ils développent une mathématique abstraite et inutile ; les lointains qu'ils scrutent avec leurs télescopes nourrissent leurs préoccupations millénaristes ou apocalyptiques. De manière générale, on peut dire qu'ils ne savent pas faire usage de leurs yeux pour voir ce qui se passe tout simplement... sous leur nez, qu'il s'agisse des

32 - Le ton ici encore en est donné par Malebranche dans ce passage de la Recherche de la vérité (op. cit. in n. 6, I, 6, p. 63-64) : "Mais quoi qu'on ne veuille pas trop s'attarder à ces choses, on a pourtant de la peine à se taire sur le mépris que les hommes font ordinairement des insectes, et des autres petits animaux qui naissent d'une matière qu'ils appellent corrompue. C'est un mépris injuste [...] Les plus petits moucherons sont des animaux aussi parfaits que les animaux les plus énormes. Les proportions de leurs membres sont aussi justes que celles des autres ; et il semble même que Dieu ait voulu leur donner plus d'ornements pour récompenser la petitesse de leur corps. Ils ont des couronnes, des aigrettes, et d'autres ajustements sur leur tête, qui effacent tout ce que le luxe des hommes peut inventer : et je puis dire hardiment que tous ceux qui ne se sont jamais servis que de leurs yeux n'ont jamais rien vu de si beau, de si juste ni même de si magnifique dans les maisons des plus grands princes, que ce que l'on voit avec des lunettes sur la tête d'une simple mouche. »

33 - Voyages, III, 2, p. 168 ; G's travels, 155. 
tromperies de leurs épouses ou, plus sérieusement, de l'ordre de la vie ordinaire, le monde commun de la cité, l'espace de la vie sociale. Cet aveuglement les rend en outre inaptes à gouverner la cité de manière noble et juste : ces savants, depuis leur île volante, exercent une domination barbare sur le continent. Ajoutons que leur science est pseudo-utilitariste, marquée par l'affirmation de I'hégémonie du théorique sur le pratique; elle se soucie peu de l'efficacité réelle des plans et projets qu'elle prescrit de façon autoritaire. L'effet est que le monde qui en résulte est le seul parmi ceux que décrit Gulliver qui présente d'horribles défauts de proportions : « [...] leurs maisons sont très mal bâties, les murs de travers, sans aucun angle droit dans un appartement ${ }^{34}$ », et tout est à I'avenant.

On a parfois jugé avec sévérité ce troisième voyage et, de manière générale, la critique swiftienne de la science qui s'y déploie. On l'estime obscurantiste ou de mauvaise foi, on signale que Swift y retrouve la veine potache du Scriblerus club, que son inspiration s'essouffle. Il me semble pourtant que ces critiques sont mal dirigées, et que les Voyages à Laputa et Balnibarbi constituent une pièce essentielle dans le projet intellectuel de Swift. II faut comprendre cependant que la critique de Swift ne porte pas pour l'essentiel sur le contenu de la science nouvelle ${ }^{35}$, la vérité de ce qu'elle découvre, mais plutôt sur le type d'attitude morale ou d'éthos qui en accompagne la pratique. Swift voit dans le New Learning une idéologie déshumanisante qui est en train de marquer de son empreinte l'ensemble des valeurs et des institutions modernes. Plus qu'à la science elle-même, il réagit donc à l'extrême valorisation sociale de ce nouvel éthos scientifique, valorisation qui se traduit notamment par le fait qu'on confie des charges politiques sensibles (comme l'administration des monnaies) à des savants, tel Newton, qui n'ont pas le sens commun ${ }^{36}$.

34 - Voyages, III, 2, p. $172 ;$ G's travels, 160.

35 - On reconnaîtra cependant que Swift laisse paraître un certain scepticisme à l'égard du progrès scientifique, notamment dans le passage savoureux de l'évocation des morts du Voyage à Glubbdubdrib. Aristote, convié devant le narrateur, déclare, après avoir avoué ses propres erreurs, que les systèmes de Descartes et de Gassendi sont dépassés et prédit le même sort au système de l'attraction de Newton. Les théories scientifiques ne seraient donc que des hypothèses incertaines qui se succèdent comme des "modes passagères ».

36 - Voir I'allusion à la distraction légendaire de Newton dans le Voyage à Laputa (Voyages, III, 2, p. 169 et note de Pons, p. 1629). Newton, qui occupait à la fin de sa vie des fonctions officielles à la direction des Monnaies, intervint de manière maladroite dans 
On perçoit bien ce que cette réaction doit aux arguments élaborés au cours du siècle précédent dans le contexte de ce qu'on a appelé la «querelle des Anciens et des Modernes ». Le jeune Swift y fut exposé très tôt par l'intermédiaire de William Temple, son tuteur, mentor, et peut-être demi-frère, qui fut, du côté des Anciens, I'un des intervenants les plus notables de la querelle ${ }^{37}$. On peut rappeler que celle-ci fut en Angleterre non pas seulement ou principalement un débat littéraire, mais un débat philosophique sur «la meilleure forme de vie », et sur ce qu'il convient, pour l'accomplir, de connaître, le type de savoir, de learning, qui l'accompagne ${ }^{38}$. Swift incontestablement participe au débat depuis ses premières oeuvres publiées, tels le Conte du tonneau ou la Bataille des livres, et se range du côté des Anciens. Dans un article important intitulé "An outline of Gulliver's travel 39 », Allan Bloom, un disciple de Leo Strauss, estime, sans doute avec raison, que les Voyages de Gulliver peuvent être considérés comme «la dernière prise de position explicite dans la fameuse querelle des Anciens et des Modernes, et peut-être l'intervention la plus importante dans ce débat fameux ». II montre en particulier que, dans les deux parties qui ne sont pas les parties «à clefs » de l'ouvrage, le Voyage à Brobdingnag et le Voyage chez les Houyhnhnms, se trouve exposée de la manière la plus positive une vision de la vie bonne et des plus saines institutions politiques que les Grecs et les Romains nous ont léguées - vision qui fait un contraste saisissant avec celle dont Gulliver lui-même, éduqué chez les Modernes anglais est imbu, et dont il finira, au terme des Voyages, par se déprendre. Les Anciens sont des géants au sens littéral, et ceux qui les fréquentent tendent aussi à grandir intérieurement. À cet égard, Bloom fait référence de manière judicieuse au thème de I'habituation perceptive rappelant ce que Gulliver explique à son second retour en Angleterre ${ }^{40}$ :

I'affaire du "demi-penny », occasion des Lettres du drapier (Dublin, 1724) où Swift dénonce l'oppression économique de I'Angleterre sur I'Irlande.

37 - Cf. son essai : William Temple, Upon Ancient and Modern learning, in Id., Miscellanea, the second part (London, 1695).

38 - Cf. sur ces questions I'ouvrage classique de Richard Foster Jones, Ancients and Moderns : A study of the back-ground of the Battle of the books (Saint Louis : Washington Univ., 1936), et pour une appréciation plus récente des enjeux idéologiques de la querelle anglaise : Joseph Levine, The Battle of the books : History and literature in the Augustan Age (Ithaca : Cornell Univ. Press, 1991), et du même, Between the Ancients and the Moderns: Baroque culture in Restoration England (New Haven : Yale Univ. Press, 1999).

39 - Édité par Joseph Cropsey dans le volume collectif Ancients and Moderns : Essays on the tradition of political philosophy in honor of Leo Strauss (New York : Basic Books, 1964).

40 - Voyages, II, 8, p. 160 ; G's travels, 144. 
" À force de voir tout au long du chemin, maisons, bêtes et gens d'une taille si minuscule, je me figurai être à Lilliput. J'avais peur d'écraser les piétons sur la route et je leur criais souvent de dégager le passage ce qui manqua de me faire rosser une fois ou deux pour mon impertinence.»

Ce qu'on peut ajouter ici est qu'il n'aurait peut-être pas même été nécessaire d'avoir été à Lilliput pour voir les Anglais comme des Lilliputiens, le séjour chez les géants ayant suffi pour créer cette perspective, et faire apparaître l'homme moderne comme un nain.

S'agissant de la question qui nous concerne plus immédiatement ici, celle de la science et de ses effets sur l'image de l'homme, il n'est pas difficile de voir où se situe la ligne de partage entre Anciens et Modernes : les premiers, tels du moins que Swift se les représente, mettaient I'homme au centre de leurs considérations, ne se préoccupant des choses extérieures que pour autant qu'elles lui étaient appropriées, utiles ou nuisibles. Ainsi les sciences chez les géants de Brobdingnag sont-elles essentiellement pratiques, tournées vers I'usage, I'agriculture, I'artisanat (ce qui fait dire d'ailleurs au voyageur Gulliver, tout enflé de préjugés modernes, que «le savoir de ce peuple est très insuffisant ${ }^{41}$ »). Les modernes en revanche, représentés par les Laputiens, plongent l'homme dans un univers dont les dimensions ne lui sont plus commensurables. L'homme n'est plus la mesure des choses puisqu'il y a des dimensions d'existence (révélées par les instruments optiques) qui excèdent complètement ses pouvoirs d'action ou de contemplation. Ainsi, les deux principes de relativité et de proportion qui, utilisés conjointement, servaient à construire la fiction optique des voyages se trouvent ici distribués de part et d'autre du partage entre Anciens et Modernes.

Ceci me conduit à une dernière considération. Swift est manifestement un admirateur des Anciens, mais sa contribution à la « querelle » dans les Voyages est assez déconcertante. L'histoire qu'il raconte, il la place délibérément dans la bouche d'un Moderne, car Gulliver en est un à l'évidence ; c'est même une sorte de savant expérimental, qui parle à la manière de Robert Boyle ou de Hooke et revendique les mêmes qualités morales, le même éthos : la sincérité, le scrupule descriptif, la curiosité désintéressée et même, comme on l'a vu au sujet du pou, le goût pour les expériences

41 - Voyages, II, 7, p. $145 ;$ G's travels, 130. 
d'anatomie salissantes. Les Voyages en outre sont construits par le moyen d'une sorte de bricolage optique et constituent dans I'ensemble une sorte d'experimentum complexe où l'individu humain sert de sujet d'expérience. On trouvait déjà le procédé qui consiste à faire de l'objet de la satire l'instrument de celle-ci dans la Bataille des livres que Swift publia en 1704 avec le Conte du tonneau. Si la Bataille est en effet une sorte d'épopée burlesque racontant le combat inégal qui met aux prises les « livres » anciens et modernes, Swift présentait son ouvrage comme l'édition moderne, "savante » et « érudite » d'un manuscrit anonyme plein $d^{\prime}$ hiatus et d'interpolations, lui adjoignant même dans la seconde édition des notes érudites découpées dans le texte que William Wotton, un célèbre défenseur des Modernes, avait écrit contre le Conte du tonneau ${ }^{42}$. La satire des philologues et des critiques, espèces de savants modernes par excellence ${ }^{43}$, emprunte donc le véhicule de la philologie et de la critique! Le même schème circulaire se retrouve dans Gulliver : un Moderne parle aux Modernes, et ne peut livrer le monde ancien que sous la forme d'un palimpseste ou d'une fiction délirante. C'est ce caractère de l'écriture swiftienne qui fait que la position de Swift, auteur et philosophe, est si difficile à assigner et qu'elle a d'ailleurs donné lieu à d'interminables conflits d'interprétation. Il me semble cependant que, dans l'adoption d'une telle méthode d'exposition, il y a bien un message, mais celui-ci est plutôt décourageant : il affirme en substance que le point de vue des Modernes est I'unique point de vue qui soit aujourd'hui à disposition, y compris lorsqu'on entend défendre I'idéal des Anciens. Ce point de vue ou cette " optique » définissent notre situation anthropologique de manière inexorable comme un horizon indépassable. Swift lui-même n'échappe donc pas à la règle. On ne peut plus être simplement et naïvement un Ancien, comme William Temple, ou son équivalent littéraire, le

42 - Cf. The Tale of a tub to which is added the Battle of the books..., $5^{\mathrm{e}}$ éd. (Londres, 1710). William Wotton publie ses Observations upon The Tale of a tub dans sa Defense of the reflections upon Ancient and Modern learning, in Answer to the objections of sir William Temple and others (Londres, 1705). Sa défense des Modernes était parue en 1694 sous le titre Reflections upon Ancient and Modern learning (Londres, 1694).

43 - Dont la figure tutélaire est le grand philologue Richard Bentley. La querelle qui I'opposa à William Temple, le mentor de Swift, au sujet de l'authenticité des Lettres de Phalaris est I'un des points d'orgue de la querelle des Anciens et des Modernes en Angleterre. Cf. The Epistles of Phalaris, [...] to which is added sir William Temple's character of the Epistles of Phalaris (London, 1699) ; Richard Bentley, A dissertation upon the Epistles of Phalaris (London, 1699). 
sage Munodi ${ }^{44}$, pensait encore pouvoir l'être, simplement en se mettant à l'écart du monde. Même les géants de Brobdingnag appartiennent au nouvel âge du monde comme en atteste le fait qu'ils sont eux aussi nostalgiques d'une époque primitive, où les hommes étaient plus robustes et la nature plus prodigue ${ }^{45}$. Compte tenu de cette situation moderne, qui est une situation de non-retour, le message swiftien des Voyages (le résultat de l'expérience) est forcément pessimiste : I'exposition à la culture et à la sagesse des Anciens, si elle peut nous guérir de l'illusion et des fantasmes de la modernité, ne nous permet pas de devenir plus heureux, elle a plutôt pour conséquence une forme de désarroi, de désocialisation, voire de folie. Ainsi en va-t-il pour le pauvre Yahoo Gulliver à son retour définitif en Angleterre : chassé de l'utopie Houyhnhnms, déchiré de n'être pas cheval parmi les chevaux, il ne peut que laisser éclater sa misanthropie et une sorte de folie hennissante.

44 - Cf. Voyages, III, 4 ; et, pour l'identification à Temple, les notes de Pons, in Swift, OEuvres, op. cit. in n. 3, 1635-1637.

45 - Voyages, II, 7, p. 147. Gulliver y évoque un petit ouvrage d'édification en vogue chez les géants, montrant « quel animal chétif, méprisable et désarmé, l'homme était de nature » et combien "la nature avait dégénéré au cours de ces derniers temps de décadence universelle ». Ces thèses sont conformes au mythe primitiviste du " déclin de la nature », dont Richard Foster Jones a montré qu'il est au cœur du tout premier état de la querelle des Anciens et des Modernes en Angleterre (op. cit. in n. 38, 22-40). 